\title{
Falling cards and flapping flags: understanding fluid-solid interactions using an unsteady point vortex model
}

Received: 30 October 2008 / Accepted: 17 February 2009 / Published online: 6 June 2009 (C) The Author(s) 2009. This article is published with open access at Springerlink.com

\begin{abstract}
A reduced-order model for the two-dimensional interaction of a sharp-edged solid body and a highReynolds number flow is presented, based on the inviscid representation of the solid's wake as point vortices with unsteady intensity. This model is applied to the fall of a rigid card in a fluid and to the flapping instability of a flexible membrane forced by a parallel flow.
\end{abstract}

Keywords Fluid-solid interaction · Point vortex · Vortex shedding

PACS 47.15.ki, 47.63.mc

\section{Introduction}

The fluttering and tumbling motions of falling cards or disks in air $[3,5,11,18,24,29]$ and the flapping of flexible membranes in parallel flows $[4,10,28,30]$ are two examples of high Reynolds number $(R e)$ fluid-solid interactions, where the full coupling between the solid and fluid dynamics leads to complex and highly unsteady behaviors. High-Re fluid-solid interactions are also essential in understanding locomotion techniques used by insects and fishes to move in fluids [26,27].

The coupling between fluid and solid equations occurs on moving boundaries whose position is itself the result of the coupled dynamics. To deal with this difficulty in numerical simulations, different techniques have been proposed, including coupled fluid-solid solvers using fitted moving grids [8,22] or immersed boundary methods [31]. The computational cost and complexity of these simulations justify parallel efforts to develop reduced-order models able to capture the relevant physics of the interaction.

In most of the applications detailed above, the solid is thin and sharp-edged. At high Re, strong vortices are shed from the corners by separation of the boundary layers. The present work describes an inviscid model for two-dimensional fluid-solid interactions based on a simplified representation of the vortical wake of the solid. The strong unsteadiness of the problem is retained. This approach differs significantly from empirical models $[2,25]$ which are based on generalization of steady results (typically the Kutta-Joukowski lift theorem) and do not contain any unsteady effect other than added inertia.

In the following sections, the general point vortex model will be presented and applied to the motion of a rigid sharp-edged body and of a flexible membrane.

Communicated by H. Aref

S. Michelin $(\bowtie) \cdot$ S. G. Llewellyn Smith

Department of Mechanical and Aerospace Engineering, Jacobs School of Engineering, UCSD, La Jolla, CA 92093-0411, USA

E-mail: smichelin@ucsd.edu

S. Michelin

Ecole Nationale Supérieure des Mines de Paris, 60-62 Boulevard Saint Michel, 75272 Paris Cedex 06, France 


\section{Coupled vortex-solid problem}

We focus here on two-dimensional problems. At sufficiently high $R e$, the pressure is dominant in the fluid forces acting on the solid and viscosity's influence is limited to the shedding of vorticity. In an inviscid model and in the absence of vortex shedding, the relative motion of the solid sharp corner and the fluid leads to a singularity in the velocity field at the solid's edge. To enforce regularity of the flow velocity in the present inviscid model, one point vortex is introduced for each shedding corner. At each time step, the intensity of this vortex is adjusted to cancel exactly the velocity singularity at the corresponding corner, rather than creating a new element of vorticity at each time step as in a continuous vortex sheet approach $[1,13,14]$. To include the irreversible nature of the vortex sheet roll-up in this model, when a vortex reaches a maximum intensity, its intensity becomes frozen and a new vortex is started from the generating corner [9].

The equation of motion of these unsteady point vortices, the Brown-Michael equation [7], is obtained by enforcing the conservation of momentum around the vortex and the associated branch cut [23]:

$$
\dot{z}_{n}+\left(z_{n}-z_{n, 0}\right) \frac{\dot{\Gamma}_{n}}{\Gamma_{n}}=\overline{\tilde{w}_{n}},
$$

where $z_{n}$ and $\Gamma_{n}$ are, respectively, the vortex position and intensity, $z_{n, 0}$ the position of the shedding corner and $\tilde{w}_{n}$ the desingularized flow velocity at the vortex position. Failing to include the corrective term in $\dot{\Gamma}_{n} / \Gamma_{n}$ would result in an unbalanced force on the branch cut linking the vortex to its generating corner [23].

Assuming the system is started from rest, the circulation at infinity must remain zero at all time. This condition allows one to solve uniquely for the flow potential given the position and intensity of the vortices as well as the solid velocity and deformation. The fluid-solid problem can be reformulated as a vortex-solid coupled problem by computing the pressure distribution on the solid (or, for a rigid body, the total force and torque applied by the fluid) as an explicit function of the vortex characteristics.

\section{Motion of a rigid body: Maxwell's problem}

For a simply connected rigid body, the time-independent geometry of the solid can be obtained by conformal mapping of a circle. The outside of the circle of radius $a$ in the mapped $\zeta$-plane is mapped onto the outside of the solid $\mathcal{C}$ in the physical $z$-plane using the mapping

$$
z=c+\mathrm{e}^{\mathrm{i} \theta} g(\zeta)
$$

with $c$ the center of mass position and $\theta$ the solid's orientation. The function $g$ entirely describes the solid geometry, and in particular, corners on $\mathcal{C}$ correspond to $g^{\prime}\left(\zeta=a \mathrm{e}^{\mathrm{i} \phi_{p}}\right)=0$ on the mapped circle boundary. The complex potential is then obtained in the mapped plane. The regularity condition and (1) can then be written as a system of equations for $\Gamma_{n}$ and $\zeta_{n}$, the vortex position in the mapped plane. Using an unsteady generalization of Blasius' theorem, the force and torque on the solid are obtained as a sum of complex integrals on the solid boundary, which can be computed exactly in terms of $c, \theta, \Gamma_{n}$ and $\zeta_{n}$, as well as their time derivatives. The reader is referred to [20] for more details and for derivation of the full equations.

The vortex model is used to study the fall of a thin card of half chord $l$ and mass $M$ per unit length in the third dimension, in a fluid of density $\rho$. This problem is also known as Maxwell's problem [19]. Experimental results have reported that above a critical $R e$ value, the broadside-on fall of the card (horizontal position) becomes unstable and either large scale fluttering or tumbling regimes are observed [11,24,29]. In a purely inviscid representation with no vortex shedding, fluid effects are limited to added inertia; from a non-zero initial angle, the solid experiences fluttering oscillations with decreasing amplitude and increasing frequency $[6,16,17]$. The asymptotic motion is a free fall in a reduced gravitational field and the broadside-on position is stable $[16,17]$. The introduction of vortex shedding destabilizes this position, and a plate released with a small angle to the horizontal first displays an unstable fluttering motion before flipping and entering rotating regimes (Fig. 1).

A similar exponential growth of the plate's oscillations was also observed in a previous study using a vortex sheet approach [14]. In both the point vortex and the vortex sheet methods, the integration eventually stops when a new element of vorticity must be created at the leading edge under small angle of attack, as such shedding conditions cannot be handled with an inviscid method $[13,14]$. This limits the validity of the method once the plate enters rotating regimes. We, therefore, concentrate on the application of this method to the initial fluttering phase where the angle of attack is large. 

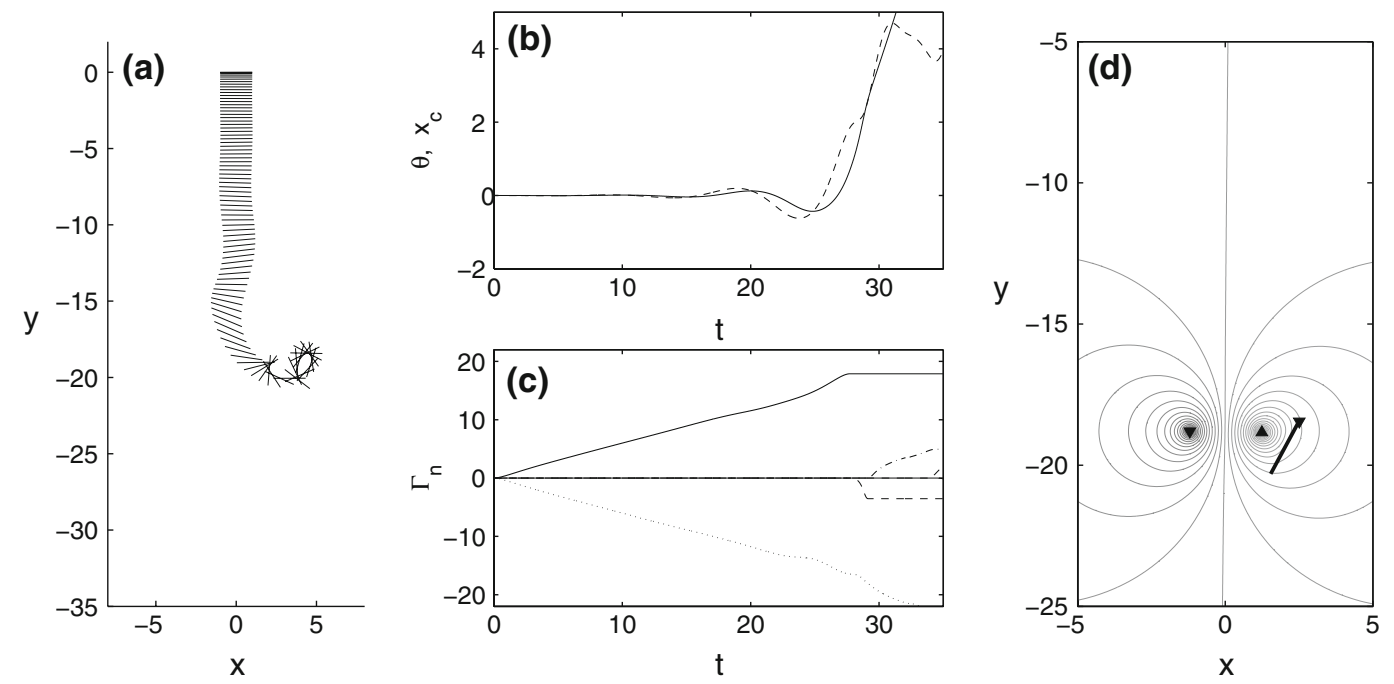

Fig. 1 Fall of a rigid card with $M / 2 \rho l^{2}=0.7$ released with an initial angle $\theta_{0}=\pi / 2048$ with the horizontal. a The position of the plate is plotted every $\Delta t=0.4$. b Orientation of the plate $\theta$ (solid) and horizontal position of the center of mass (dashed). c Intensities of the successive vortices shed by the plate's corners. d Snapshot of the streamlines for $t=28$, shortly before the flip of the plate. Positive (resp. negative) vortices are represented by upward- (resp. downward-) pointing triangles. All quantities have been non-dimensionalized using the half-chord $l$, the fluid density $\rho$ and gravity $g$ as reference quantities

The low-order representation of the wake using point vortices provides some physical insight on the destabilization process. Several vortices are shed once the amplitude of the plate's angular motion becomes large. However, during the initial small-amplitude fluttering regime, the wake can be represented to a good approximation as a horizontal pair of vortices of opposite intensities. In the absence of the plate, the momentum of these vortices is vertical and oriented downward. The plate (because of the image vorticity and its tilted orientation) slightly deflects this vortex momentum in the direction opposite to the plate's lateral velocity. During one half oscillation, horizontal momentum is transferred to the vortices in the opposite direction to the plate motion, thereby creating an accelerating force on the solid and destabilizing the broadside-on position [20].

\section{Motion of a flexible body: flapping of a flexible membrane}

We consider here the motion of a two-dimensional flexible membrane forced by an imposed parallel flow $U_{\infty}$ of density $\rho . L$ is the length of the membrane in the streamwise direction, $\rho_{s}$ its mass per unit area and $B$ its bending rigidity per unit length in the third dimension. All quantities are non-dimensionalized using $L, U_{\infty}$ and $\rho$ as reference quantities. Though still valid in theory, conformal mapping techniques become very difficult to use because of the time-dependence of the mapping. To study the flow over an infinitely thin membrane, the solid is instead represented as a bound-vorticity distribution $\kappa(s, t)$ (with $0 \leq s \leq 1$ the curvilinear coordinate) solution of a singular integral equation obtained from the normal flow condition on the membrane $[1,13,21]$ :

$$
\frac{1}{2 \pi} \int_{0}^{1} \operatorname{Re}\left[\frac{\mathrm{e}^{\mathrm{i} \theta\left(s_{0}\right)}}{\zeta\left(s_{0}\right)-\zeta(s)}\right] \kappa \mathrm{d} s=\operatorname{Im}\left[\mathrm{e}^{\mathrm{i} \theta}\left(U_{\infty}+\frac{1}{2 \pi \mathrm{i}} \sum \frac{\Gamma_{n}}{\zeta-z_{n}}-\frac{\partial \bar{\zeta}}{\partial t}\right)\right],
$$

with $\zeta(s)$ the position of the membrane and $\theta(s)$ its orientation.

The complex flow velocity is obtained as the superposition of $U_{\infty}$ and the contribution from the vorticity (bound-vorticity distribution $\kappa$ for the solid, discrete vortices $\left(z_{n}, \Gamma_{n}\right)$ for the wake), and the pressure jump is obtained from Bernoulli's theorem:

$$
[p]^{ \pm}\left(s_{0}\right)=\int_{0}^{s_{0}} \dot{\kappa} \mathrm{d} s+\kappa u_{p}
$$



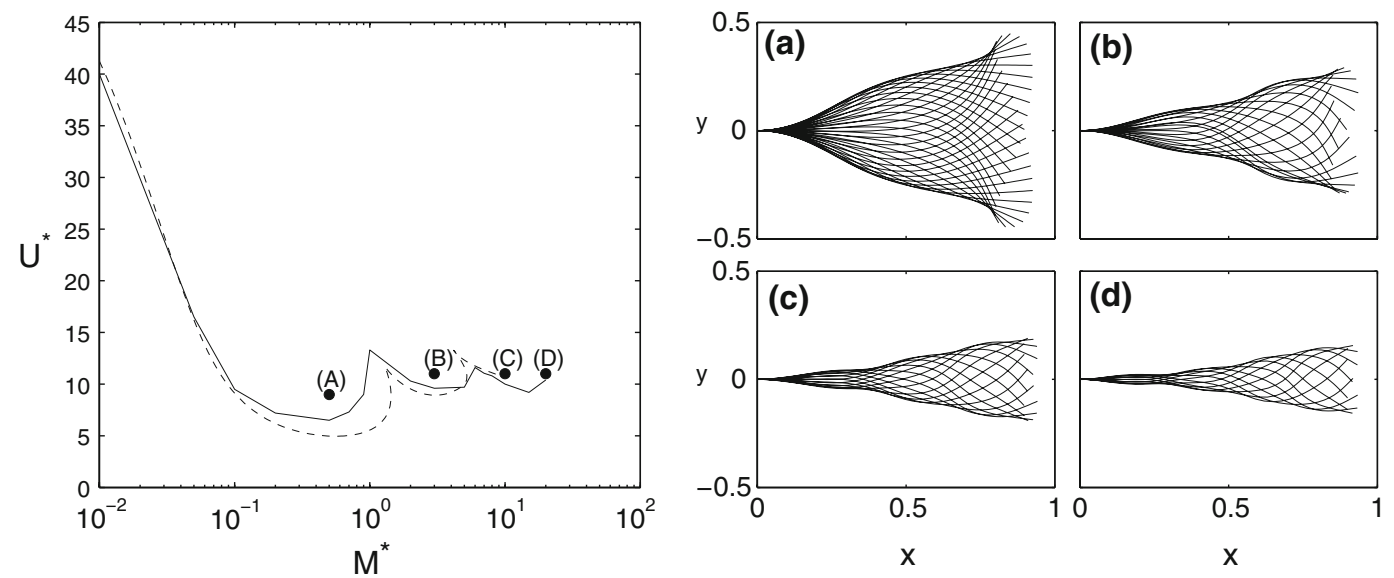

Fig. 2 (Left) Critical stability curve of the rest position of the membrane in the ( $M^{*}, U^{*}$ )-plane (solid) compared to the linear stability analysis results in $[10,15]$ (dashed). (Right) Shape of the flapping mode observed for (a) $M^{*}=0.5$ and $U^{*}=9$, (b) $M^{*}=3$ and $U^{*}=11$, (c) $M^{*}=10$ and $U^{*}=11$ and (d) $M^{*}=20$ and $U^{*}=11$. The position of the membrane is plotted every $\Delta t=0.08$

where $u_{p}$ is the principal value of the relative tangential fluid velocity. The internal solid dynamics is described using a large displacement inextensible Euler-Bernoulli beam model with clamped-free boundary conditions and is solved together with (3)-(4) using a second-order semi-implicit finite difference scheme and Chebyshev spectral methods [21].

The problem is characterized by two non-dimensional parameters $(\mu, \eta)$ with $\mu$ the inertia ratio and $\sqrt{\eta}$ the time-scale ratio of the aerodynamic destabilization to the restoring elastic force (or alternatively $\left(M^{*}, U^{*}\right)$ with $U^{*}$ the imposed flow non-dimensionalized by the flag properties):

$$
\mu=\frac{1}{M^{*}}=\frac{\rho_{s}}{\rho L}, \quad \eta=\frac{1}{M^{*} U^{* 2}}=\frac{B}{\rho L^{3} U_{\infty}^{2}} .
$$

We are interested in the stability of the (straight) state of rest of the flag. The system is started from rest and a small vertical perturbation is added to the parallel flow at infinity. For a given mass ratio, the flag state of rest becomes unstable when the elastic response is too slow compared to the pressure forcing (small $\eta$ or large $U^{*}$ ). The critical curve $U_{c}\left(M^{*}\right)$ is obtained as the minimum value of $U^{*}$ above which the flag does not return to its flat position after being perturbed, and compares well with previous linear stability results (see Fig. 2). Above the critical curve and for intermediate values of $U^{*}$, a flapping regime develops with a structure determined by the most linearly unstable mode, as observed in experimental results [10]. If the flow velocity is increased further, a transition to a chaotic regime is observed. The reader is referred to [21] for more details on the model and flag instability results.

The model can be generalized to study the coupled flapping of $N$ membranes. For $N=2$, and given $M^{*}$ and $U^{*}$, in-phase (resp. out-of-phase) flapping modes and wake structures are observed for small (resp. large) separation $D / L$ (Fig. 3) in agreement with experimental observations [12,30].

\section{Conclusions}

The unsteady point vortex method presented here allows one to reduce significantly the computational complexity and cost of two-dimensional fluid-solid interactions, while still retaining most of the physical effects, in particular the strong unsteadiness of vortex shedding. It allows one to obtain qualitative (destabilization of a falling card by the shed vorticity) and quantitative (stability curve and flapping modes shape of a flexible flag) insights into several important fluid-solid interactions.

The method is particularly suited to describe the shedding of vorticity from the solid's trailing edge or at high angle of attack, as in both cases the vortex wake is advected away from the body. As for the vortex sheet approach $[13,14]$, the point vortex method's ability to describe leading-edge shedding at small angle of attack is limited, as viscous effects and interaction of the newly shed vortex with the boundary layer on the solid are expected to become dominant. We, therefore, restricted our application of this method to the initial fluttering 
(a)

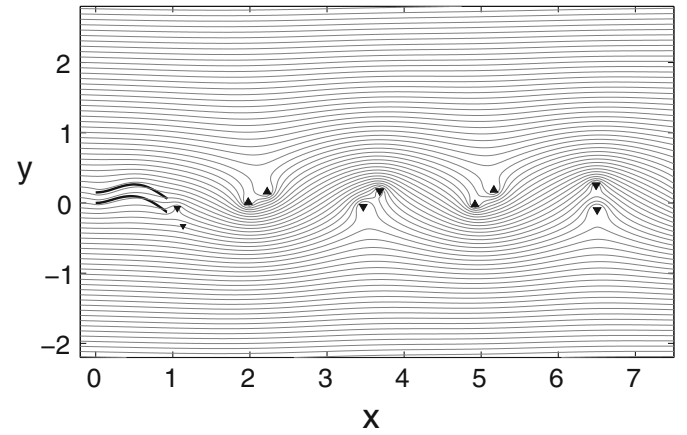

(b)

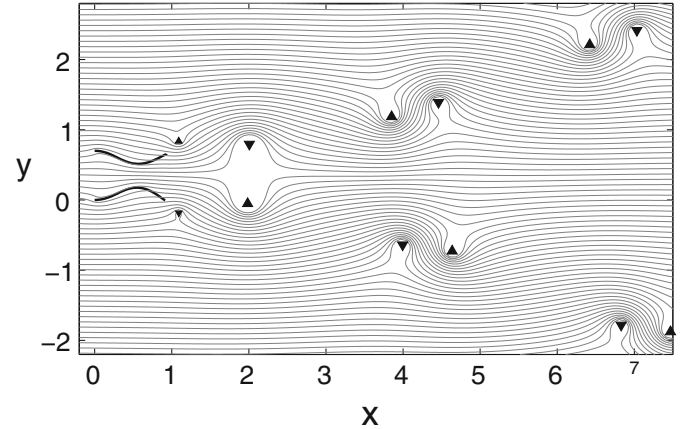

Fig. 3 Instantaneous streamlines in the flapping regime with two identical parallel membranes with $M^{*}=0.5$ and $U^{*}=8$, positioned at a distance $\mathbf{a} D=0.15 \mathrm{~L}$ and $\mathbf{b} D=0.7 \mathrm{~L}$. Positive (resp. negative) wake vortices are represented with upward- (resp. downward-) pointing triangles. For small distances, the membranes flap in phase and the wake is similar to the one-membrane problem. For larger distances, the membranes flap in opposition of phase, and the wake consists of vortex pairs arranging on two symmetric diverging tracks

regime of the falling card or flapping membranes shedding vortices from the trailing edge only. In the latter case, the effect of leading-edge shedding is expected to be negligible in a first approximation, as the angle of attack remains small at all time.

By representing the wake as a discrete distribution of point vortices, this method provides a theoretical tool to understand the fluid forces as transfer of momentum to the vortex wake. Because of its reduced computational cost (compared to direct numerical simulations or even the vortex sheet approach), this reduced-order model can be of particular interest for problems where the cost of other techniques is prohibitive (e.g. optimization in locomotion studies).

Acknowledgements This work was supported by NSF award CTS-0133978 and the Human Frontier Science Program Research Grant RGY 0073/2005.

Open Access This article is distributed under the terms of the Creative Commons Attribution Noncommercial License which permits any noncommercial use, distribution, and reproduction in any medium, provided the original author(s) and source are credited.

\section{References}

1. Alben, S., Shelley, M.J.: Flapping states of a flag in an inviscid fluid: bistability and the transition to chaos. Phys. Rev. Lett. 100, 074301 (2008)

2. Andersen, A., Pesavento, U., Wang, Z.J.: Analysis of transitions between fluttering, tumbling and steady descent of falling cards. J. Fluid Mech. 541, 91-104 (2005)

3. Andersen, A., Pesavento, U., Wang, Z.J.: Unsteady aerodynamics of fluttering and tumbling plates. J. Fluid Mech. 541, 65-90 (2005)

4. Balint, T.S., Lucey, A.D.: Instability of a cantilevered flexible plate in viscous channel flow. J. Fluids Struct. 20, 893-912 (2005)

5. Belmonte, A., Eisenberg, H., Moses, E.: From flutter to tumble: inertial drag and Froude similarity in falling paper. Phys. Rev. Lett. 81, 345-348 (1998)

6. Borisov, A.V., Kozlov, V.V., Mamaev, I.S.: Asymptotic stability and associated problems of dynamics of falling rigid body. Reg. Chaotic Dyn. 12, 531-565 (2007)

7. Brown, C.E., Michael, W.H.: Effect of leading edge separation on the lift of a delta wing. J. Aero. Sci. 21, 690-694, 706 (1954)

8. Connell, B.S.H., Yue, D.K.P.: Flapping dynamics of a flag in uniform stream. J. Fluid Mech. 581, 33-67 (2007)

9. Cortelezzi, L., Leonard, A.: Point vortex model of the unsteady separated flow past a semi-infinite plate with transverse motion. Fluid Dyn. Res. 11, 263-295 (1993)

10. Eloy, C., Lagrange, R., Souilliez, C., Schouveiler, L.: Aeroelastic instability of a flexible plate in a uniform flow. J. Fluid Mech. 611, 97-106 (2008)

11. Field, S.B., Klaus, M., Moore, M.G., Nori, F.: Chaotic dynamics of falling disks. Nature 388, 252-254 (1997)

12. Jia, L.B., Li, F., Yin, X.Z., Yin, X.Y.: Coupling modes between two flapping filaments. J. Fluid Mech. 581, 199-220 (2007)

13. Jones, M.A.: The separated flow of an inviscid fluid around a moving plate. J. Fluid Mech. 496, 405-441 (2003)

14. Jones, M.A., Shelley, M.J.: Falling cards. J. Fluid Mech. 540, 393-425 (2005)

15. Kornecki, A., Dowell, E.H., O'Brien, J.: On the aeroelastic instability of two-dimensional panels in uniform incompressible flow. J. Sound Vib. 47, 163-178 (1976) 
16. Kozlov, V.V.: Heavy rigid body falling in an ideal fluid. Mech. Solids 24, 9-17 (1989)

17. Mahadevan, L.: Tumbling of a falling card. C. R. Acad. Sci. Ser. IIb 323, 729-736 (1996)

18. Mahadevan, L., Ryu, W.S., Samuel, A.D.T.: Tumbling cards. Phys. Fluids 11, 1-3 (1999)

19. Maxwell, J.C.: On a particular case of the decent of a heavy body in a resisting medium. Camb. Dublin Math. J. 9, 145-148 (1854)

20. Michelin, S., Llewellyn Smith, S.G.: An unsteady point vortex method for coupled fluid-solid problems. Theor. Comp. Fluid Dyn. (2009, in press). doi:10.1007/s00162-009-0096-7

21. Michelin, S., Llewellyn Smith, S.G., Glover, B.J.: Vortex shedding model of a flapping flag. J. Fluid Mech. 617, 1-10 (2008)

22. Pesavento, U., Wang, Z.J.: Falling paper: Navier-Stokes solutions, model of fluid forces, and center of mass elevation. Phys. Rev. Lett. 93, 144501 (2004)

23. Rott, N.: Diffraction of a weak shock with vortex generation. J. Fluid Mech. 1, 111-128 (1956)

24. Smith, E.H.: Autorotating wings: an experimental investigation. J. Fluid Mech. 50, 513-534 (1971)

25. Tanabe, Y., Kaneko, K.: Behavior of a falling paper. Phys. Rev. Lett. 73, 1372-1375 (1994)

26. Triantafyllou, M.S., Triantafyllou, G.S., Yue, D.K.P.: Hydrodynamics of fishlike swimming. Annu. Rev. Fluid Mech. 32 , $33-53(2000)$

27. Wang, Z.J.: Dissecting insect flight. Annu. Rev. Fluid Mech. 37, 183-210 (2005)

28. Watanabe, Y., Suzuki, S., Sugihara, M., Sueoka, Y.: An experimental study of paper flutter. J. Fluids Struct. 16, 529-542 (2002)

29. Willmarth, W.W., Hawk, N.E., Harvey, R.L.: Steady and unsteady motions and wakes of freely falling disks. Phys. Fluids 7, 197-208 (1964)

30. Zhang, J., Childress, S., Libchaber, A., Shelley, M.: Flexible filaments in a flowing soap film as a model for one-dimensional flags in a two-dimensional wind. Nature 408, 835-839 (2000)

31. Zhu, L., Peskin, C.: Simulation of flapping flexible filament in a flowing soap film by the immersed boundary method. J. Comp. Phys. 179, 452-468 (2002) 